\title{
Fungal periprosthetic joint infection following total elbow arthroplasty: a case report and review of the literature
}

\author{
Cory A. Kwong ${ }^{1 *}$, Shannon K. T. Puloski ${ }^{2}$ and Kevin A. Hildebrand ${ }^{3}$
}

\begin{abstract}
Background: With improving surgical techniques for total elbow arthroplasty clinical outcomes have improved and its utilization continues to increase. Despite these advances, complication rates remain as high as $24 \%$. Of these complications periprosthetic joint infection is one of the most common and morbid. The rheumatoid elbow remains a leading indication for total elbow arthroplasty. Patients with this condition frequently require immunosuppressive therapy, which places them at higher risk of both typical and atypical infections.

Case presentation: We present the case of a persistent, late-onset periprosthetic joint infection in a total elbow arthroplasty of a 64-year-old Caucasian woman with severe refractory rheumatoid arthritis. The offending pathogen, Aspergillus terreus, is previously unreported in the arthroplasty literature and grew concurrently with coagulasenegative staphylococcus. Eradication of the fungal and bacterial agents involved resection arthroplasty, serial debridement, and multiple courses of intravenous and oral antimicrobial therapy. Two attempts at reimplantation arthroplasty failed to eliminate the infection and our patient ultimately required definitive resection arthroplasty.

Conclusions: Arthroplasty in the rheumatoid elbow confers with it a high complication rate. Inflammatory disease and immunosuppressive drugs combined with the subcutaneous anatomy of the elbow contribute to the risk of infection. Fungal periprosthetic joint infection in the rheumatoid patient presents both diagnostic and therapeutic challenges. Fungal growth should always be treated and requires organism-specific antimicrobials in conjunction with surgical debridement. More literature is needed to determine the optimal treatment regimen for this devastating complication.
\end{abstract}

Keywords: Total elbow arthroplasty, Periprosthetic joint infection, Aspergillus terreus, Infection, Rheumatoid arthritis, Fungal, Revision, Resection arthroplasty

\section{Background}

With improvements in outcomes of total elbow arthroplasty (TEA) [1], the procedure has become increasingly more common. Rates of TEA utilization in the United States doubled over a 14-year period to a rate of 0.96 per 100,000 [2]. Despite advances in technique and hardware components, complication rates remain high in comparison to other joint arthroplasties. A systematic review of the literature from 1992 to 2009 estimated that the significant complication rate was as high as $24.3 \%$

\footnotetext{
* Correspondence: cakwong87@gmail.com

${ }^{1}$ Orthopaedic Surgery Resident PGY-3, Section of Orthopedic Surgery,

Department of Surgery, University of Calgary, Health Sciences Centre, 3330

Hospital Drive NW, Calgary, AB T2N 4N1, Canada

Full list of author information is available at the end of the article
}

[1]. Of these complications, periprosthetic joint infections (PJI) are one of the more common and most devastating, and have been estimated to occur in 5-8\% of patients [3]. The treatment of PJI is well described in arthroplasty literature and carries with it significant morbidity. However, the literature on PJI in TEA is still limited and, to the best of our knowledge, there are no published reports of fungal infection following TEA. The existing literature concerning fungal PJI of the other joints mostly consists of case series and consensus statements. Here we present the complex case of persistent, late-onset Aspergillosis of a total elbow arthroplasty in a patient with severe refractory rheumatoid arthritis. 


\section{Case presentation}

A right-handed, 64-year-old Caucasian woman presented for reimplantation left TEA. She had had a left total elbow resection arthroplasty in April 2014 due to a fungal PJI. Her past medical history was significant for a 41year history of severe refractory rheumatoid arthritis involving multiple joints and cervical spine. She had failed multiple medical therapies including disease-modifying antirheumatic drugs and biologics, and was treated intermittently with prednisone for flares.

The original DePuy Pritchard TEA (DePuy, Warsaw, IN, United States) was implanted in 1995. It had performed well until June 2007 when she developed left elbow pain and fevers and received a liner exchange in well-fixed implants (Fig. 1). The TEA was retained until she required resection arthroplasty in July 2011 after repeat debridement and antimicrobial regimens failed to resolve a draining sinus due to a coagulase-negative staphylococcus (CONS) infection. The implant was replaced with a vancomycin-impregnated cement spacer. Intraoperative cultures grew CONS for which she was treated with cefazolin for 8 weeks. Two months postoperatively the spacer was removed but tissue cultures remained positive for polymicrobial infection including CONS and Enterobacter cloacae. Extended antimicrobials consisted of 6 weeks of ciprofloxacin and vancomycin. In December of 2011 reimplantation was attempted but abandoned when intraoperative frozen sections showed $>30$ white blood cells per high-powered field (WBC/hpf). Definitive tissue cultures grew new
Aspergillus terreus only, which was thought to be a contaminant after growing on only one of six fungal cultures.

In February 2012, she was off of all immunosuppressives and systemically well. The elbow was healed and free of any drainage. Her C-reactive protein (CRP) level was $8.7 \mathrm{mg} / \mathrm{L}$ and erythrocyte sedimentation rate (ESR) was 29. Reimplantation surgery was undertaken using a similar method reported by LeBlanc et al. 2012 [4]; an allograft-prosthetic composite was used consisting of a long-stemmed cemented Coonrad-Morrey TEA (Zimmer) and tibial allograft on the humeral side (Fig. 2). Intraoperatively there was no concern for infection, but final cultures grew scant CONS and Aspergillus terreus and histologic samples were nonspecific. She was treated as a mixed fungal and bacterial PJI and treated with 8 weeks of intravenous voriconazole and vancomycin. The implant was retained.

In the following 6 months she healed and was doing well until a rheumatoid flare. She was put on a trial of abatacept and shortly after developed a fluctuant mass around the lateral distal humerus. An aspiration of the collection grew Aspergillus terreus and the abatacept was stopped. Blood work showed a CRP level of 10.4 and an ESR of 50. Over the course of the next 7 months, she would undergo eight more serial aspirates of the recurrent collection with only the first specimen growing Aspergillus terreus. A bone scan showed no increased uptake, but repeat radiographs showed the presence of an insufficiency fracture on the ulnar side (Fig. 3).

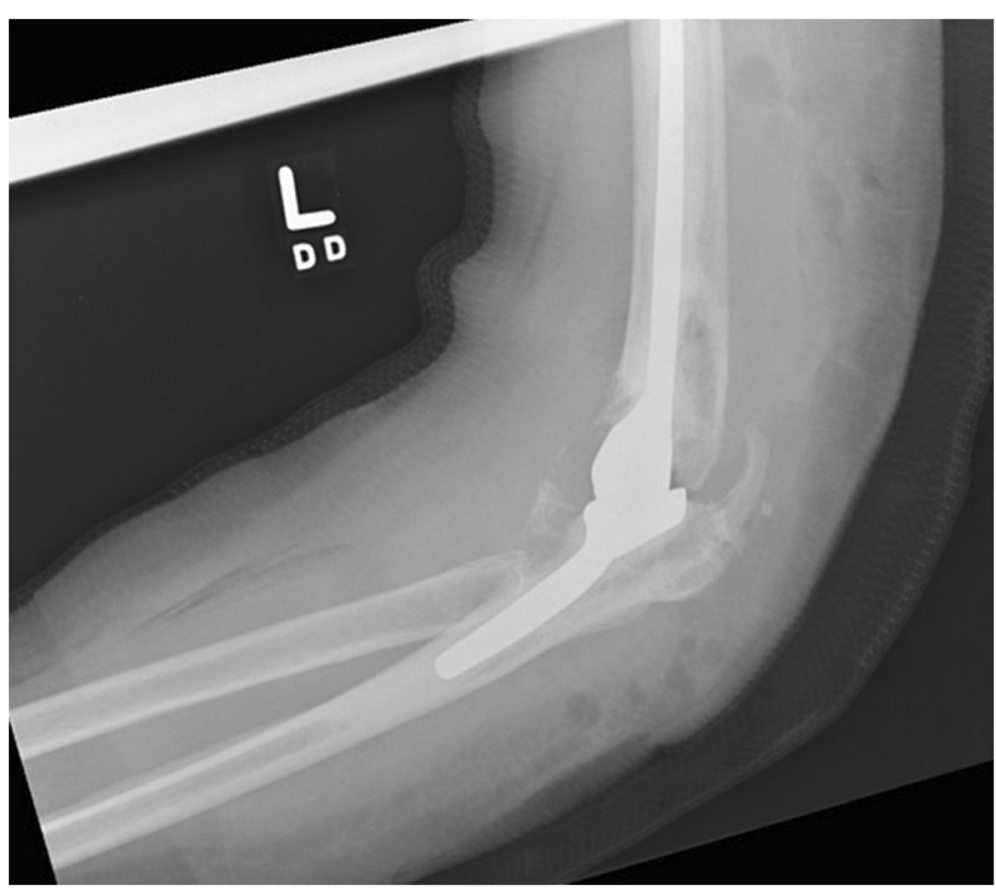

Fig. 1 Left total elbow arthroplasty showing well-fixed components 

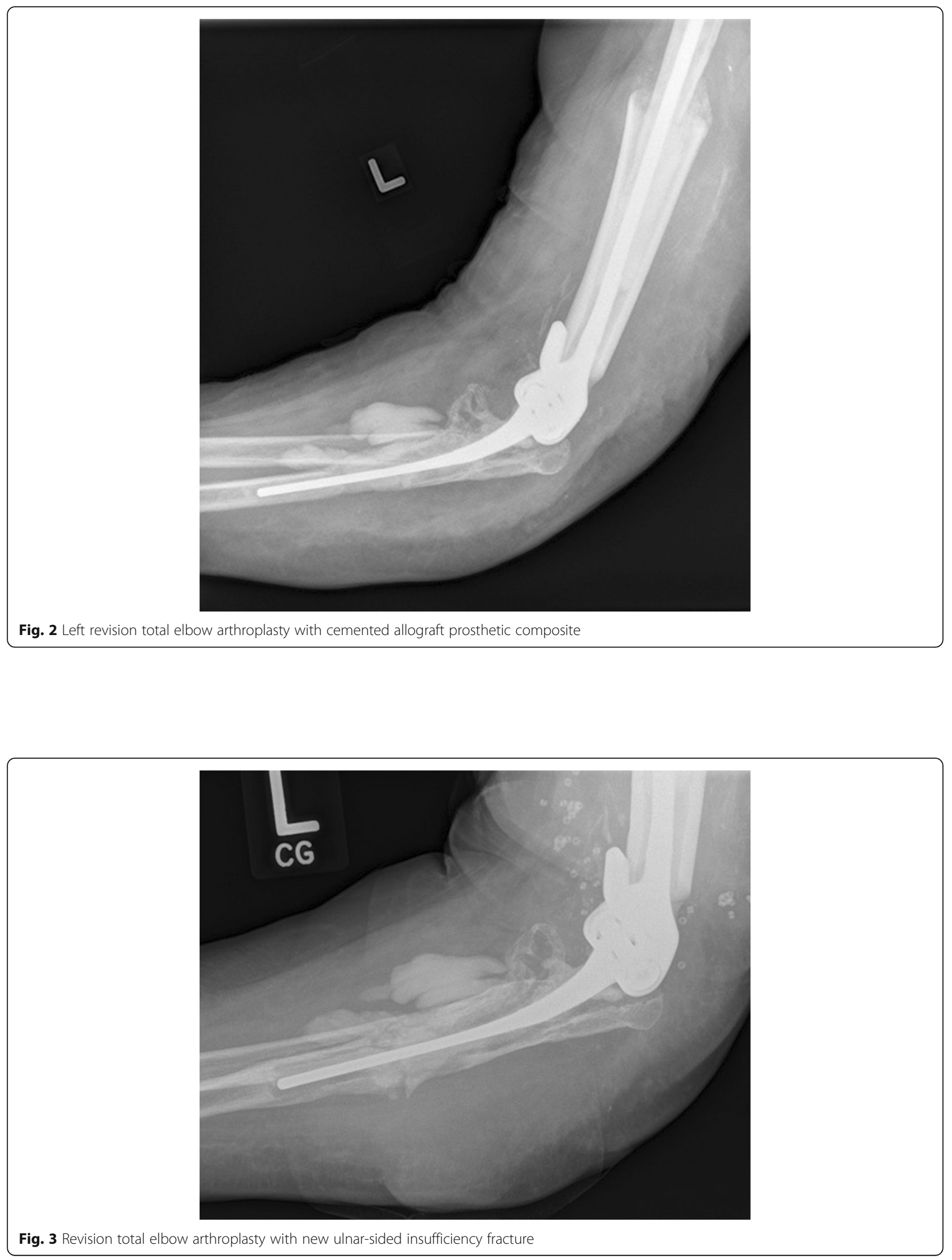
During this time she remained off of antimicrobial treatment in an attempt to identify the offending organism.

With failure to control Aspergillosis of the left TEA with combined serial (two) operative debridements and medical management, a second resection arthroplasty was performed in April 2014. Aspergillus terreus grew on one of three cultures but no bacterial growth was identified. Postoperative antimicrobial therapy consisted of caspofungin and cefazolin for 8 weeks, followed by cephalexin and a short trial of oral voriconazole, which was stopped due to gastrointestinal intolerance and transaminitis.

Eleven months post left TEA resection arthroplasty for chronic Aspergillosis, she was systemically well and had been off of antimicrobials for 5 months. A physical examination revealed a left flail elbow, she was neurologically intact with good function of the hand and a well-healed posteriorly based scar. There were no signs of infection or inflammation. Her most recent laboratory test results showed a WBC count of $6.2 \times 10 \mathrm{E} 9 / \mathrm{L}$, a CRP level of $2.2 \mathrm{mg} / \mathrm{L}$ and an ESR of 25, all within normal limits. The last positive tissue culture was from the resection arthroplasty in April 2014 and no further specimens had been collected. Plain radiographs showed a significant osseous defect of the proximal ulna and distal humerus (Fig. 4). A bone scan and white blood cell scan showed no definitive evidence of ongoing infection.
In March 2015, after consultation with infectious diseases and local orthopedic colleagues, our patient and surgeons elected to proceed with a second attempt at reimplantation arthroplasty. Due to extensive bone loss, the reimplantation was performed using a cemented distal humerus endoprosthesis and long-stemmed ulna component (Biomet SRS/Discovery TEA; Zimmer Biomet) (Figs. 5 and 6). Multiple tissue specimens collected intraoperatively were negative for fungal and bacterial growth.

Unfortunately, after a brief symptom-free period she developed a recurrent sinus over the tip of the olecranon and recurrent CONS was confirmed. Our patient has since undergone definitive resection arthroplasty. No further fungal infection was identified and no further surgery is planned.

\section{Discussion}

Fungal infection in elbow arthroplasty

Only 1\% of PJIs are of fungal origin [5], and because of this rarity, the literature is limited to a few small case series and consensus statements mostly based on hip and knee arthroplasty. Since relatively few TEAs are performed compared to hip and knee arthroplasties, the incidence of fungal PJI in TEA is unknown [6]. The most common organisms in fungal PJI are Candida species [7]. Aspergillus fumigatus and Aspergillus niger have also

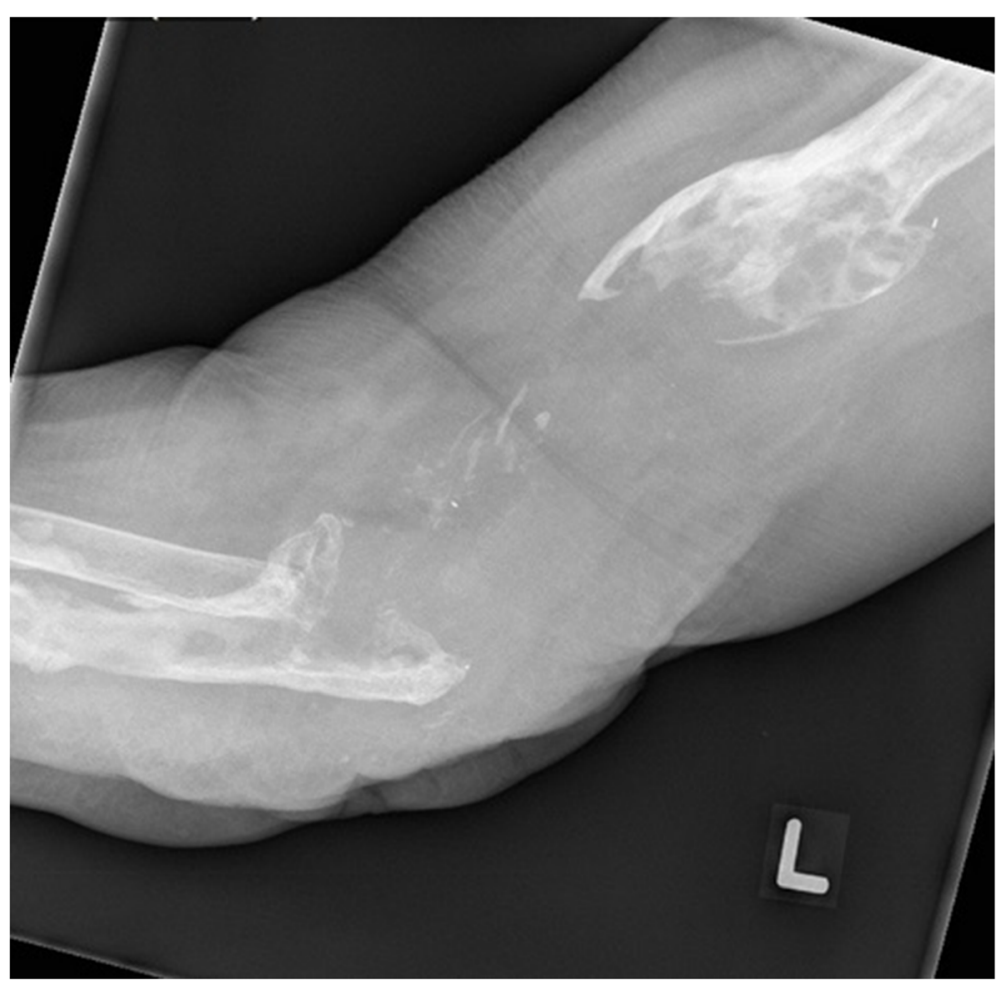

Fig. 4 Left elbow resection arthroplasty 


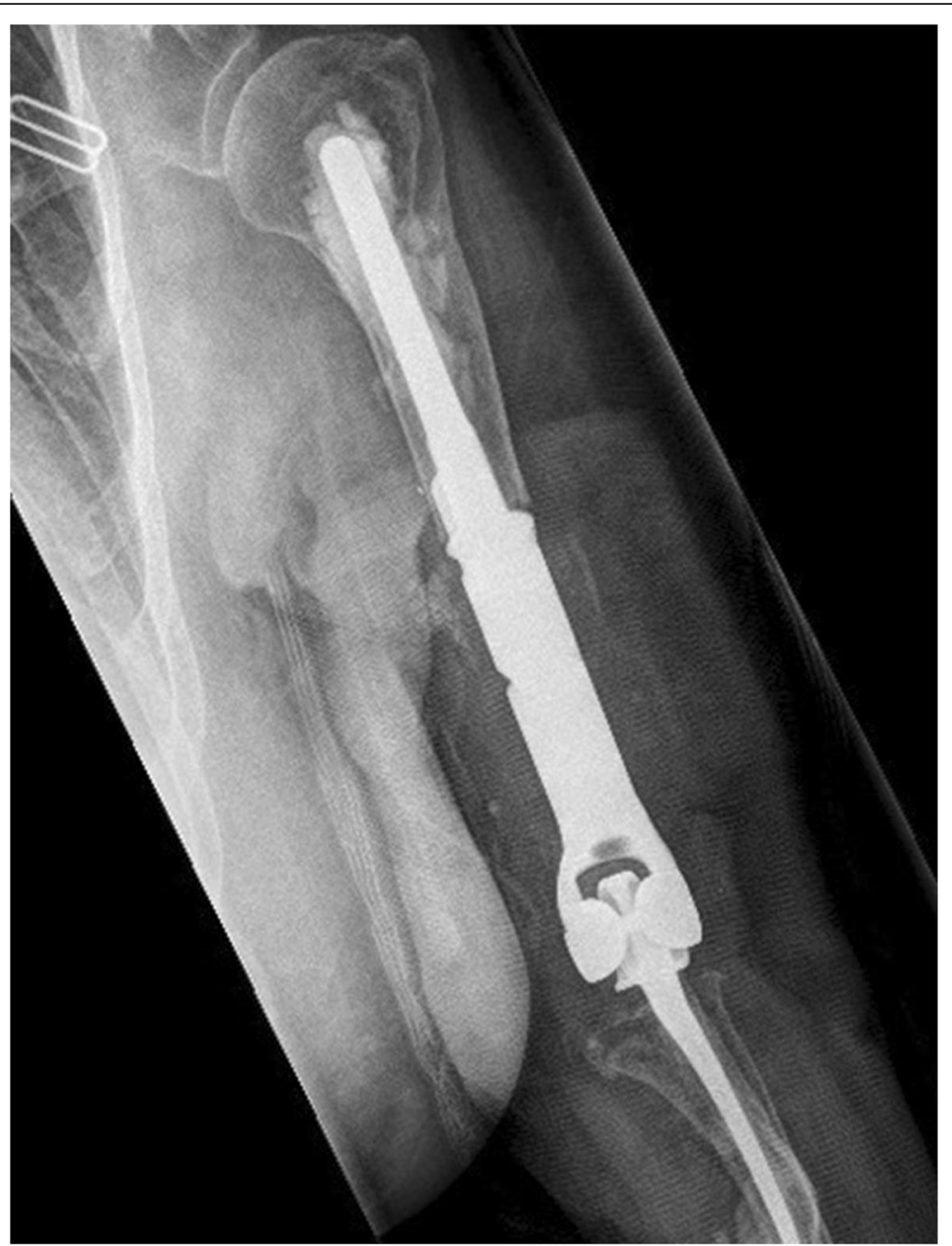

Fig. 5 Left revision total elbow arthroplasty anteroposterior view

been reported in the arthroplasty literature in small numbers [8] but, to the best of our knowledge, there have been none for Aspergillus terreus.

Many patients requiring TEA have pre-existing rheumatoid arthritis (RA). It has been proposed that the high complication rate of TEA in patients with RA could be attributed to a combination of poor bone stock, minimal posterior soft tissue coverage, and immunodeficiency [9]. In RA, either the pathologic process itself causes the immunodeficiency, or the pharmacology used to treat it [10]. Like in our case, many of these patients will be at higher risk of atypical infections, which can potentially lead to catastrophic morbidity

\section{Diagnosis of fungal infection in elbow arthroplasty}

Fungal PJIs are often hard to diagnose and can be complicated by comorbidities and concurrent or previous bacterial infection [10]. Regardless, diagnosis begins with a thorough history and physical examination similar to any presentation of PJI. Diagnostic imaging including plain radiographs should always be obtained, but advanced three-dimensional imaging and nuclear medicine tests have not been recommended for routine use in the diagnosis of PJIs [11]. Serologic markers are unable to distinguish between causative organisms and synovial fluid rarely identifies fungal pathogens [10]. As a result of this, special attention must be given to specimen collection, as routine cultures may show no growth in the setting of a high clinical suggestion. To improve diagnostic yield, serial joint aspirations and multiple intraoperative specimens from diagnostic or therapeutic procedures are essential to help establish the causative organism [12, 13]. Fungal cultures should be plated on fungal selective media (for example, Sabourad dextrose) and growth can take up to 4 weeks [5]. When cultures do yield fungal organisms, results are still often misinterpreted. In a 2013 systematic review of fungal PJIs, Kuiper et al. found that fungal growth was initially 


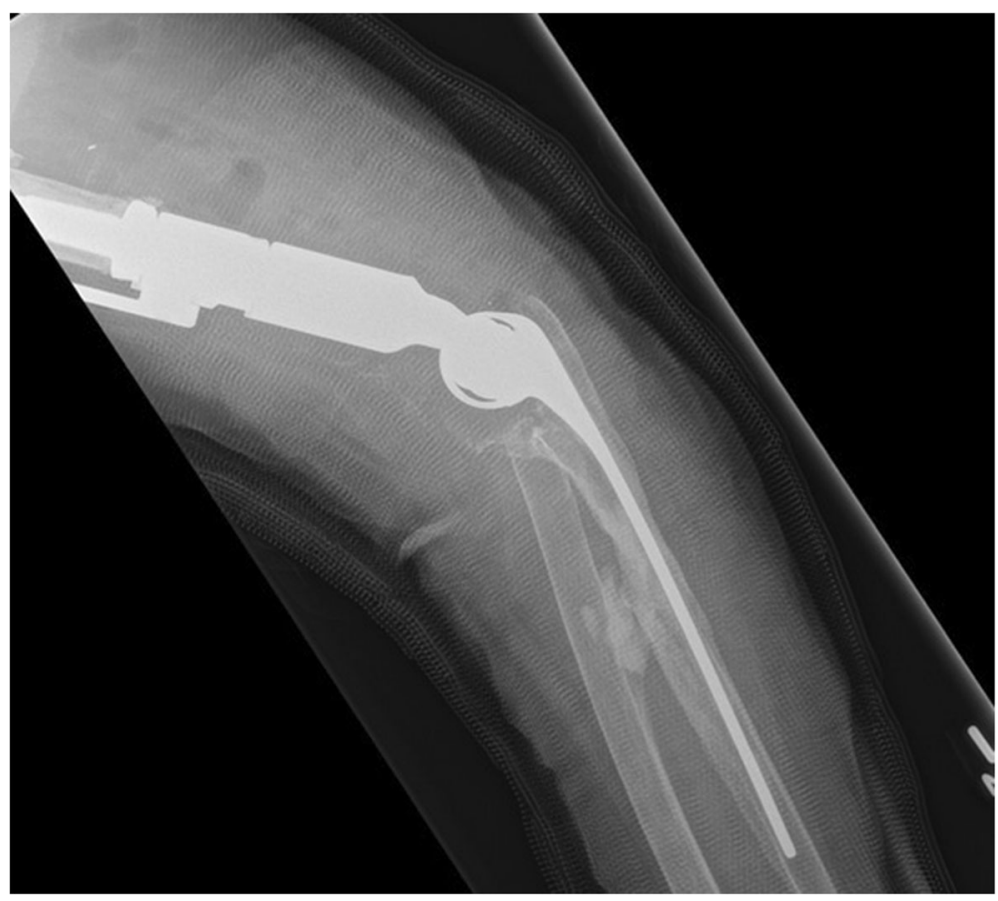

Fig. 6 Left revision total elbow arthroplasty lateral view

considered contamination in $21 \%$ of cases. They concluded that any fungal species grown should thus be considered a pathogen [7].

In this case, the underlying RA and history of bacterial PJI in the same joint complicated the diagnosis. Serologic markers including ESR and CRP were difficult to interpret in the context of severe RA. The previous and intermittent presence of CONS from aspirates and surgical specimens not only confounded organism-specific therapy but also significantly increased the patient's baseline risk for a fungal PJI [12]. Initial suggestion of Aspergillus spp. as a contaminant may have led to delayed time to antifungal treatment and premature second-stage reimplantation with the first revision. In retrospect, a histologic evaluation of $\mathrm{WBC} / \mathrm{hpf}$ at the time of reimplantation in February 2012 may have detected continued inflammation and reconsideration of the Aspergillus spp. as a contaminant. However, while frozen histologic section has been shown to be highly specific at $93.1 \%$, it is only $51.3 \%$ sensitive [14]. Additionally, its utility in both fungal infections and patients with underlying arthropathies is still poorly defined [15].

\section{Eradication of fungal infection in elbow arthroplasty}

Fungal PJIs are not only difficult to diagnose but are also thought to be challenging to treat. Standardized protocols for the treatment of PJI have been produced by the Infectious Diseases Society of America (IDSA) but have not been customized for fungal PJI, let alone fungal PJI in TEA. The optimal treatment of PJI consists of both medical and surgical intervention $[3,11,12]$.

Medical management includes systemic antifungals prior to reimplantation, and debate still exists surrounding the use of antifungal-impregnated spacers owing to a lack of evidence [12]. The IDSA currently recommends between 4 and 6 weeks of organism-specific intravenous or highly bioavailable oral antimicrobial therapy following resection arthroplasty, but does not distinguish between bacterial and fungal organisms. As was seen in this case, prolonged use of antifungals raises the risk of systemic toxicity and poses a challenge when considering length of therapy [5]. Cheung et al. used a similar antimicrobial treatment regimen in their experience of 29 bacterial PJIs of TEAs. Medical management in twostage revisions included tobramycin/vancomycin-impregnated cement spacers and 6 weeks of organismspecific intravenous antibiotics. None of their causative organisms were fungal [16].

Surgical options include resection arthroplasty, oneand two-staged revision, arthrodesis, and amputation. A 2015 systematic review of 45 fungal infections in total knee arthroplasty recommended a two-staged approach as the gold standard. This consisted of resection arthroplasty with or without antibiotic-impregnated cement spacers, followed by delayed reimplantation. As an initial surgical intervention, the failure rate was approximately $30 \%$ [12]. Cheung et al. reported on 29 TEA reimplantations for bacterial PJI from 1976 to 2003. They showed a 
similar failure rate of $28 \%$, with a 3 -year survival of $77 \%$ and 8-year survival of only $48 \%$ [16]. The necessity for removal of all original hardware may be attributable to the ability of fungi, including Aspergillus spp., to form hardy biofilms overlying prostheses [17]. A 1998 retrospective study on PJI in TEA showed that four of four Staphylococcus epidermidis PJIs, a known biofilm-forming species, failed irrigation and debridement with hardware retention, compared to two of eight failures for Staphylococcus aureus PJIs [18].

\section{Conclusions}

Fungal PJIs in TEA are different from both bacterial PJIs in TEA, and fungal PJIs in hip and knee arthroplasty. Host risk factors including inflammatory disease and immunosuppressive drugs combined with the subcutaneous anatomy of the elbow are possible risk factors that could contribute to the higher complication and infection rates.

It has been hypothesized that the introduction of modern antirheumatic drugs has contributed to a decrease in the utilization of joint surgery in rheumatoid arthritis [17]. Even though improved medical treatment in RA may have contributed to a decreased incidence of rheumatoid elbow as an indication for TEA [6], patients who do undergo the procedure for this indication may be at higher risk of atypical PJIs caused by fungi.

A lack of literature on this rare but morbid complication left the responsible team without a precedent on which to base treatment. In this case, fungal infection of a TEA for rheumatoid elbow proved to be an extremely difficult complication to manage and caused considerable morbidity to the patient. Owing to the rarity of this complication, demonstrated by the lack of literature, future cases will pose similar therapeutic dilemmas. If presented with a similar case, the authors advise orthopedic surgeons and infectious disease specialists not to attribute positive fungal cultures to contamination, especially when it may delay treatment in an immunocompromised host. As was seen in this case, fungal infection may persist despite evidence of preoperative sterility. In patients with a history of recurrent infected TEA, surgeons should be wary of the morbidity of multiple attempts at revision endoprosthetic reconstruction in favor of earlier resection arthroplasty. It is our hope that continued follow-up and future reports will provide insight into a successful strategy for treatment of fungal infections in TEA.

\section{Acknowledgements}

None.

\section{Funding}

The authors declare that they did not receive any specific funding for this work.

Availability of data and materials

Not applicable.

\section{Authors' contributions}

CK conducted the chart review, literature search, and prepared drafts of the manuscript for this case report. $\mathrm{KH}$ and SP were the treating surgeons; they contributed to editing and drafting of the manuscript. All authors reviewed and approved the final manuscript for submission.

\section{Competing interests}

The authors declare that they have no competing interests.

\section{Consent for publication}

Written informed consent was obtained from the patient for publication of this case report and any accompanying images. A copy of the written consent is available for review by the Editor-in-Chief of this journal.

\section{Ethics approval and consent to participate}

At the University of Calgary under the Tri-Council Policy Statement: Ethical Conduct for Research Involving Humans (TCPS 2) of the Conjoint Health Research Ethics Board, the publication of case reports does not require ethics approval. http://www.ucalgary.ca/research/researchers/ethics-compliance/ chreb\#quickset-field_collection_quicktabs_4.

\section{Author details}

${ }^{1}$ Orthopaedic Surgery Resident PGY-3, Section of Orthopedic Surgery, Department of Surgery, University of Calgary, Health Sciences Centre, 3330 Hospital Drive NW, Calgary, AB T2N 4N1, Canada. ${ }^{2}$ Section of Orthopedic Surgery, Department of Surgery, University of Calgary, Health Sciences Centre, 3330 Hospital Drive NW, Calgary, ABT2N 4N1, Canada. ${ }^{3}$ Department of Surgery, University of Calgary, Health Sciences Centre, 3330 Hospital Drive NW, Calgary, ABT2N 4N1, Canada.

Received: 4 October 2016 Accepted: 11 December 2016 Published online: 21 January 2017

\section{References}

1. Voloshin I, Schippert DW, Kakar S, Kaye EK, Morrey BF. Complications of total elbow replacement: a systematic review. J Shoulder Elbow Surg. 2011;20(1):158-68.

2. Singh JA, Ramachandran R. Sex differences in characteristics, utilization, and outcomes of patient undergoing total elbow arthroplasty: a study of the US nationwide inpatient sample. Clin Rheumatol. 2016;35(3):723-31.

3. Kim JM, Mudgal CS, Konopka JF, Jupiter JB. Complications of total elbow arthroplasty. J Am Acad Orthop Surg. 2011;19(6):328-39.

4. Leblanc J, Puloski S, Hildebrand K. Interprosthetic humeral fracture revision using a tibial allograft total elbow prosthetic composite in a patient with hemophilia A: a case report. J Med Case Reports. 2012;6:319.

5. Bariteau JT, Waryasz GR, McDonnell M, Fischer SA, Hayda RA, Born CT. Fungal osteomyelitis and septic arthritis. J Am Acad Orthop Surg. 2014;22(6):390-401.

6. Gay DM, Lyman S, Do H, Hotchkiss RN, Marx RG, Daluiski A. Indications and reoperation rates for total elbow arthroplasty: an analysis of trends in New York State. J Bone Joint Surg Am. 2012;94(2):110-7.

7. Kuiper JW, van den Bekerom MP, van der Stappen J, Nolte PA, Colen S. 2stage revision recommended for treatment of fungal hip and knee prosthetic joint infections. Acta Orthop. 2013;84(6):517-23.

8. Yilmaz M, Mete B, Ozaras R, Kaynak G, Tabak F, Tenekecioglu Y, Ozturk R. Aspergillus fumigatus infection as a delayed manifestation of prosthetic knee arthroplasty and a review of the literature. Scand J Infect Dis. 2011; 43(8):573-8

9. Cook C, Hawkins R, Aldridge 3rd JM, Tolan S, Krupp R, Bolognesi M. Comparison of perioperative complications in patients with and without rheumatoid arthritis who receive total elbow replacement. J Shoulder Elbow Surg. 2009;18(1):21-6.

10. Azzam K, Parvizi J, Jungkind D, Hanssen A, Fehring T, Springer B, Bozic K, Della Valle C, Pulido L, Barrack R. Microbiological, clinical, and surgical features of fungal prosthetic joint infections: a multi-institutional experience. J Bone Joint Surg Am. 2009;91 Suppl 6:142-9.

11. Osmon DR, Berbari EF, Berendt AR, Lew D, Zimmerli W, Steckelberg JM, Rao N, Hanssen A, Wilson WR. Infectious Diseases Society of America. Diagnosis and management of prosthetic joint infection: clinical practice guidelines by the Infectious Diseases Society of America. Clin Infect Dis. 2013;56(1):e1-e25.

12. Jakobs O, Schoof B, Klatte TO, Schmidl S, Fensky F, Guenther D, Frommelt L, Gehrke T, Gebauer M. Fungal periprosthetic joint infection in total knee arthroplasty: a systematic review. Orthop Rev (Pavia). 2015;7(1):5623. 
13. Gebauer M, Frommelt L, Achan P, Board TN, Conway J, Griffin W, Heidari N, Kerr G, McLaren A, Nelson SB, Nijhof M, Zahar A. Management of fungal or atypical periprosthetic joint infections. J Arthroplasty. 2014;29(2 Suppl):112-4.

14. Ahmadi S, Lawrence TM, Morrey BF, Sanchez-Sotelo J. The value of intraoperative histology in predicting infection in patients undergoing revision elbow arthroplasty. J Bone Joint Surg Am. 2013;95(21):1976-9.

15. Della Valle C, Parvizi J, Bauer TW, DiCesare PE, Evans RP, Segreti J, Spangehl M, Watters III WC, Keith M, Turkelson CM. Diagnosis of periprosthetic joint infections of the hip and knee. J Am Acad Orthop Surg. 2010;18(12):760-70.

16. Cheung EV, Adams RA, Morrey BF. Reimplantation of a total elbow prosthesis following resection arthroplasty for infection. J Bone Joint Surg Am. 2008;90(3):589-94

17. Coad BR, Kidd SE, Ellis DH, Griesser HJ. Biomaterials surfaces capable of resisting fungal attachment and biofilm formation. Biotechnol Adv. 2014; 32(2):296-307.

18. Yamaguchi K, Adams RA, Morrey BF. Infection after total elbow arthroplasty. J Bone Joint Surg Am. 1998;80(4):481-91.

Submit your next manuscript to BioMed Central and we will help you at every step:

- We accept pre-submission inquiries

- Our selector tool helps you to find the most relevant journal

- We provide round the clock customer support

- Convenient online submission

- Thorough peer review

- Inclusion in PubMed and all major indexing services

- Maximum visibility for your research

Submit your manuscript at www.biomedcentral.com/submit
Biomed Central 